\title{
Scaling of the superfluid density in high-temperature superconductors
}

\author{
C. C. Homes* S. V. Dordevic, T. Valla, and M. Strongin \\ Department of Physics, Brookhaven National Laboratory, Upton, New York 11973
}

(Dated: July 23, 2018)

\begin{abstract}
A scaling relation $\rho_{s} \simeq 35 \sigma_{d c} T_{c}$ has been observed in the copper-oxide superconductors, where $\rho_{s}$ is the strength of the superconducting condensate, $T_{c}$ is the critical temperature, and $\sigma_{d c}$ is the normal-state dc conductivity close to $T_{c}$. This scaling relation is examined within the context of a clean and dirty-limit BCS superconductor. These limits are well established for an isotropic BCS gap $2 \Delta$ and a normal-state scattering rate $1 / \tau$; in the clean limit $1 / \tau \ll 2 \Delta$, and in the dirty limit $1 / \tau>2 \Delta$. The dirty limit may also be defined operationally as the regime where $\rho_{s}$ varies with $1 / \tau$. It is shown that the scaling relation $\rho_{s} \propto \sigma_{d c} T_{c}$ is the hallmark of a BCS system in the dirty-limit. While the gap in the copper-oxide superconductors is considered to be $d$-wave with nodes and a gap maximum $\Delta_{0}$, if $1 / \tau>2 \Delta_{0}$ then the dirty-limit case is preserved. The scaling relation implies that the copper-oxide superconductors are likely to be in the dirty limit, and that as a result the energy scale associated with the formation of the condensate is scaling linearly with $T_{c}$. The $a-b$ planes and the $c$ axis also follow the same scaling relation. It is observed that the scaling behavior for the dirty limit and the Josephson effect (assuming a BCS formalism) are essentially identical, suggesting that in some regime these two effects may be viewed as equivalent. This raises the possibility that electronic inhomogeneities in the copper-oxygen planes may play an important role in the nature of the superconductivity in the copper-oxide materials.
\end{abstract}

PACS numbers: 74.25.Gz, 74.25.-q, 74.72.-h, 72.15.Lh

\section{INTRODUCTION}

Scaling laws express a systematic and universal simplicity among complex systems in nature. For example, such laws are of enormous significance in biology, where the scaling relation between body mass and metabolic rate spans 21 orders of magnitude 1.2 Scaling relations are equally important in the physical sciences. Since the discovery of superconductivity at elevated temperatures in copper-oxide materials ${ }^{3}$ there has been considerable effort to find trends and correlations between the physical quantities, as a clue to the origin of the superconductivity. $\stackrel{4}{*}$ One of the earliest patterns that emerged was the linear scaling of the superfluid density $\rho_{s} \propto 1 / \lambda^{2}$ (where $\lambda$ is the superconducting penetration depth) in the copper-oxygen planes of the hole-doped materials with the superconducting transition temperature $T_{c}$. This is referred to as the Uemura relation, and it works reasonably well for the underdoped materials. However, it does not describe very underdoped, optimally doped (i.e., $T_{c}$ is a maximum), overdoped,, 8.9 or electron-doped materials. ${ }^{10,11}$ A similar attempt to scale $\rho_{s}$ with the dc conductivity $\sigma_{d c}$ was only partially successful 12 We have recently demonstrated that the scaling relation $\rho_{s} \propto \sigma_{d c} T_{c}$ may be applied to a large number of high-temperature superconductors, regardless of doping level or type, nature of disorder, crystal structure, or direction (parallel or perpendicular to the copper-oxygen planes) 13 The optical values of $\rho_{s}\left(T \ll T_{c}\right)$ and $\sigma_{d c}\left(T \gtrsim T_{c}\right)$ within the $a$ - $b$ planes have been determined for a large number of copper-oxide superconductors, as well as the bismuth-oxide material $\mathrm{Ba}_{1-x} \mathrm{~K}_{x} \mathrm{BiO}_{3}$; the results are shown as a linear plot in Fig. 1] In this representation, the underdoped points near the origin tend to lie rather close together, and the $c$-axis points which are not shown would be visible only as a single point slightly below the underdoped data. While there is some scatter in the data, a linear trend is clearly visible, although it has been suggested that there is some deviation from this behavior in the extremely-overdoped materials, 14 When plotted as a log-log plot in Fig. 2 the linear trend is more apparent, and indicates that within the error the points may be described by the scaling relation $\rho_{s} \simeq 35 \sigma_{d c} T_{c}$ (in this instance both sides of the equation possess the same units, so that the constant is dimensionless). In addition, the elemental BCS superconductors $\mathrm{Nb}$ and $\mathrm{Pb}$ (without any special regards to preparation) are also observed to follow this scaling relation reasonably well.

\section{EXPERIMENT}

The values for $\sigma_{d c}$ and $\rho_{s}$ shown in Table I have been obtained almost exclusively from reflectance measurements from which the complex optical properties have been determined through a Kramers-Kronig analysis. 15 The dc conductivity has been extrapolated from the real part of the optical conductivity $\sigma_{d c}=\sigma_{1}(\omega \rightarrow 0)$ at $T \gtrsim T_{c}$. For $T \ll T_{c}$, the response of the dielectric function to the formation of a condensate is expressed purely by the real part of the dielectric function $\epsilon_{1}(\omega)=\epsilon_{\infty}-\omega_{p s}^{2} / \omega^{2}$, which allows the strength of the condensate to be calculated from $\omega_{p s}^{2}=-\omega^{2} \epsilon_{1}(\omega)$ in the $\omega \rightarrow 0$ limit. Here, $\omega_{p s}^{2}=4 \pi n_{s} e^{2} / m^{*}$ is the square of the superconducting plasma frequency and $\rho_{s} \equiv \omega_{p s}^{2}$, and $\epsilon_{\infty}$ is the high-frequency contribution to the real part of the dielectric function. The strength of the condensate may 


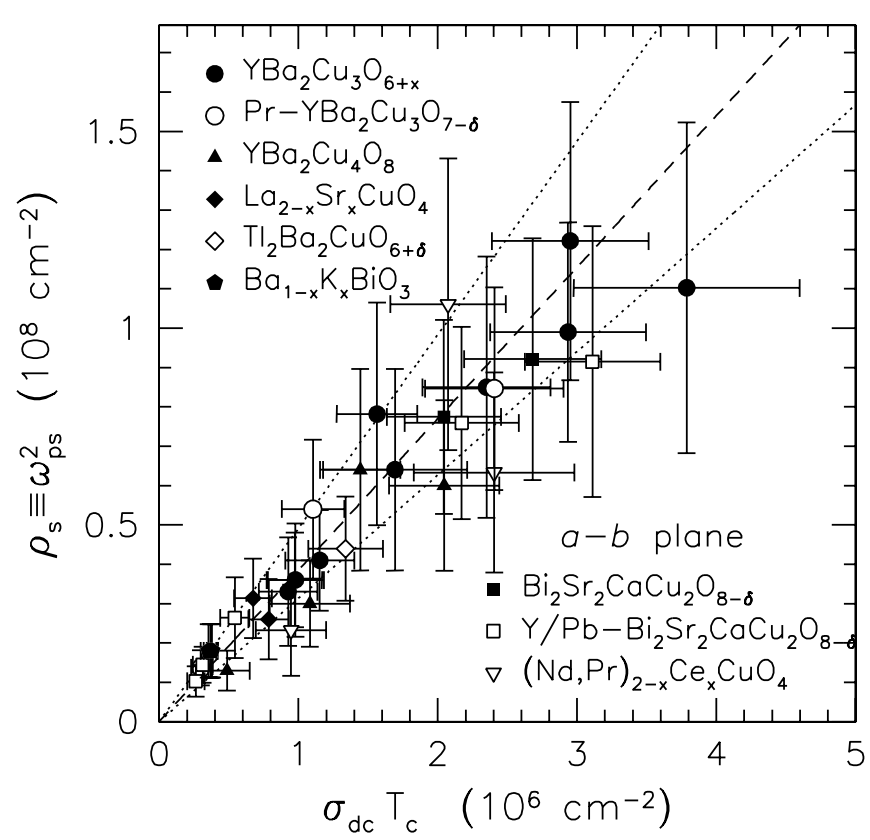

FIG. 1: The superfluid density $\rho_{s}$ vs $\sigma_{d c} T_{c}$ for the $a-b$ planes of the hole-doped copper-oxide superconductors for pure and Pr-doped $\mathrm{YBa}_{2} \mathrm{Cu}_{3} \mathrm{O}_{6+x}$ (Refs. 12202122 23); $\mathrm{YBa}_{2} \mathrm{Cu}_{4} \mathrm{O}_{8}$ (Ref. 21); pure and Y/Pb-doped $\mathrm{Bi}_{2} \mathrm{Sr}_{2} \mathrm{CaCu}_{2} \mathrm{O}_{8+\delta}$ (Refs. 23, 24 25); underdoped $\mathrm{La}_{2-x} \mathrm{Sr}_{x} \mathrm{CuO}_{4}$ (Ref. 26); $\mathrm{Tl}_{2} \mathrm{Ba}_{2} \mathrm{CuO}_{6+\delta}$ (Ref. 27); electron-doped (Nd,Pr) ${ }_{2-x} \mathrm{Ce}_{x} \mathrm{CuO}_{4}$ (Refs. 1028 , 29 ) and the bismate material $\mathrm{Bi}_{1-x} \mathrm{~K}_{x} \mathrm{BiO}_{3}$ (Ref. 30). Within error, all the points may be described by a single (dashed) line, $\rho_{s} \simeq 35 \sigma_{d c} T_{c}$; the upper and lower dotted lines, $\rho_{s} \simeq$ $44 \sigma_{d c} T_{c}$ and $28 \sigma_{d c} T_{c}$ respectively, represent approximately the spread of the data.

also be estimated by tracking the changes in the spectral weight above and below $T_{c}$, where the spectral weight is defined as $\frac{16}{\underline{16}} N(\omega, T)=(120 / \pi) \int_{0^{+}}^{\omega} \sigma_{1}\left(\omega^{\prime}, T\right) d \omega^{\prime}$. The condensate may be calculated from the shift in the spectral weight $\rho_{s}=N_{n}-N_{s}$, where $N_{n}=N\left(\omega, T \simeq T_{c}\right)$, and $N_{s}=N_{s}\left(\omega, T \ll T_{c}\right)$. This is the Ferrell-GloverTinkham sum rule which tracks changes in the optical conductivity $\sigma_{1}(\omega)$ above and below $T_{c}$ due to the formation of a condensate at zero frequency 17,18 These two different techniques typically yield nearly identical values for $\rho_{s}$; an exception exists in the underdoped materials along the $c$ axis, where it has been suggested that there is missing spectral weight. 19

\section{DISCUSSION}

A deeper understanding of the scaling relation as it relates to both the elemental superconductors and the copper-oxide materials may be obtained from an examination of the spectral weight above and below $T_{c}$ in relation to the normal-state scattering rate and the superconducting energy gap. When $\mathrm{Nb}$ is in the dirty limit, it follows the $\rho_{s} \propto \sigma_{d c} T_{c}$ relation, but in the clean limit there is a deviation from this linear behavior. (This result will be explored in more detail shortly.) The terms

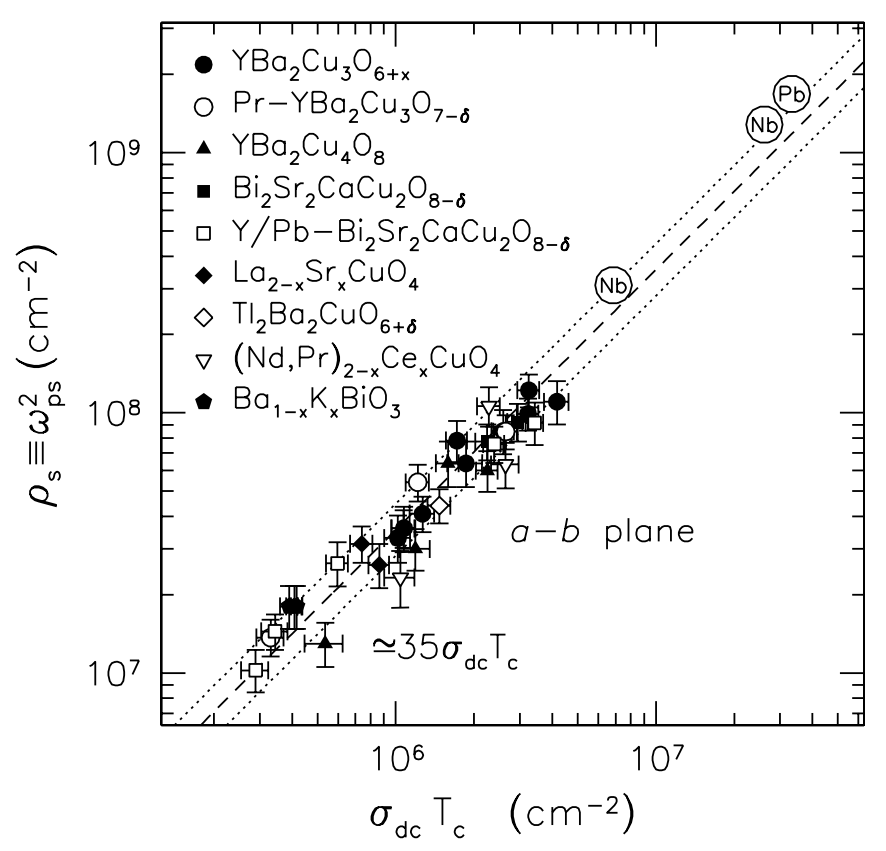

FIG. 2: The log-log plot of the superfluid density $\rho_{s}$ vs $\sigma_{d c} T_{c}$ for the $a-b$ planes of the hole-doped copper-oxide superconductors shown in Fig. 11 The dashed and dotted lines are shown in this figure are the sames lines that were shown in Fig. 1 The points for $\mathrm{Nb}$ and $\mathrm{Pb}$, indicated by the atomic symbols, also fall close to the dotted line, $\rho_{s} \simeq 35 \sigma_{d c} T_{c}$ (Refs. 32 33).

"clean" and "dirty" originate from the comparison of the isotropic BCS energy gap $2 \Delta$ with the normal-state scattering rate $1 / \tau$; the clean limit is taken as $1 / \tau \ll 2 \Delta$, while the dirty limit is $1 / \tau>2 \Delta$. The clean and dirty limits may also be expressed as $l \gg \xi_{0}$ and $l<\xi_{0}$, respectively, where $l$ is the quasiparticle mean-free path and $\xi_{0}$ is the BCS coherence length; because $l \propto \tau$ and $\xi_{0} \propto 1 / \Delta$, this is equivalent to the previous statement $\underline{31}$ The use of these definitions depends on having accurate values for $1 / \tau$ and $\Delta$. In general, BCS superconductors have relatively low values for $T_{c}$, thus $1 / \tau$ is assumed to have little temperature dependence close to the superconducting transition. This assumption may be tested by suppressing $T_{c}$ through the application of a magnetic field in excess of the upper critical field $\left(\mathrm{H}_{c 2}\right)$ and examining the transport properties, which typically reveal little temperature dependence in $1 / \tau$ below the zero-field value of $T_{c}$. The application of the the clean and dirtylimit picture to the copper-oxide superconductors is complicated by both the high critical temperature, and the superconducting energy gap which is thought to be $d$ wave in nature and momentum dependent $\left(\Delta_{k}\right)$, containing nodes 34.35 The high value for $T_{c}$ suggests that $1 / \tau$ may still have a significant temperature dependence close to $T_{c}$. In the normal state the scattering rate is often observed to be rather large, scaling linearly with temperature,$\frac{36}{3}$ and is presumed to be dominated by inelastic processes. Indeed, below $T_{c}$ the quasiparticle scattering rate in the cuprates is observed to decrease by 
TABLE I: The critical temperature $T_{c}$, dc conductivity $\sigma_{d c} \equiv \sigma_{1}(\omega \rightarrow 0)$ just above $T \gtrsim T_{c}$, plasma frequency of the condensate $\omega_{p s}$ and penetration depth $\lambda_{a b}$ for $T \ll T_{c}$, for light polarized in the $a$ - $b$ planes for a variety of single and double-layer copperoxygen high-temperature superconductors. Values for $\mathrm{Ba}_{1-x} \mathrm{~K}_{x} \mathrm{BiO}_{3}$ as well as several elemental superconductors have also been included.

\begin{tabular}{|c|c|c|c|c|c|c|}
\hline Material & Note & (Ref.) & $T_{c}(\mathrm{~K})$ & $\sigma_{d c}\left(\Omega^{-1} \mathrm{~cm}^{-1}\right)$ & $\omega_{p s}\left(\mathrm{~cm}^{-1}\right)$ & $\lambda_{a b}(\mu \mathrm{m})$ \\
\hline $\mathrm{YBa}_{2} \mathrm{Cu}_{3} \mathrm{O}_{6.95}$ & $a, b$ & $(20)$ & 70 & $4400 \pm 500$ & $5750 \pm 600$ & 0.276 \\
\hline $\mathrm{YBa}_{2} \mathrm{Cu}_{3} \mathrm{O}_{6.95}$ & $a, b$ & $(20)$ & 80 & $6500 \pm 600$ & $8840 \pm 800$ & 0.180 \\
\hline $\mathrm{YBa}_{2} \mathrm{Cu}_{3} \mathrm{O}_{6.95}$ & $a, b$ & $(20)$ & 85 & $9200 \pm 900$ & $9220 \pm 900$ & 0.172 \\
\hline $\mathrm{YBa}_{2} \mathrm{Cu}_{3} \mathrm{O}_{6.95}$ & $a, b$ & $(20)$ & 93.5 & $10500 \pm 1000$ & $11050 \pm 800$ & 0.144 \\
\hline $\mathrm{YBa}_{2} \mathrm{Cu}_{3} \mathrm{O}_{6.60}$ & $\mathrm{c}$ & $(21.22)$ & 59 & $6500 \pm 600$ & $6400 \pm 500$ & 0.248 \\
\hline $\mathrm{YBa}_{2} \mathrm{Cu}_{3} \mathrm{O}_{6.95}$ & $\mathrm{c}$ & $(21.22)$ & 93.2 & $10500 \pm 900$ & $9950 \pm 700$ & 0.159 \\
\hline $\mathrm{YBa}_{2} \mathrm{Cu}_{3} \mathrm{O}_{7-\delta}$ & $\mathrm{b}$ & $(23)$ & 92 & $8700 \pm 900$ & $9200 \pm 700$ & 0.172 \\
\hline $\mathrm{Pr}-\mathrm{YBa}_{2} \mathrm{Cu}_{3} \mathrm{O}_{7-\delta}$ & $\mathrm{b}$ & $(23)$ & 40 & $2500 \pm 300$ & $3700 \pm 300$ & 0.430 \\
\hline $\mathrm{Pr}-\mathrm{YBa}_{2} \mathrm{Cu}_{3} \mathrm{O}_{7-\delta}$ & $\mathrm{b}$ & (23) & 75 & $4900 \pm 500$ & $7350 \pm 600$ & 0.216 \\
\hline $\mathrm{YBa}_{2} \mathrm{Cu}_{4} \mathrm{O}_{8}$ & $\mathrm{c}$ & (21) & 80 & $6000 \pm 600$ & $8000 \pm 800$ & 0.198 \\
\hline $\mathrm{Bi}_{2} \mathrm{Ca}_{2} \mathrm{SrCu}_{2} \mathrm{O}_{8+\delta}$ & $\mathrm{c}$ & $(24)$ & 90 & $11500 \pm 900$ & $9565 \pm 900$ & 0.166 \\
\hline $\mathrm{Bi}_{2} \mathrm{Ca}_{2} \mathrm{SrCu}_{2} \mathrm{O}_{8+\delta}$ & $\mathrm{b}$ & (23) & 91 & $9800 \pm 800$ & $9600 \pm 800$ & 0.165 \\
\hline $\mathrm{Bi}_{2} \mathrm{Ca}_{2} \mathrm{SrCu}_{2} \mathrm{O}_{8+\delta}$ & $\mathrm{b}$ & (23) & 85 & $8500 \pm 800$ & $8710 \pm 700$ & 0.182 \\
\hline $\mathrm{Y} / \mathrm{Pb}-\mathrm{Bi}_{2} \mathrm{Ca}_{2} \mathrm{SrCu}_{2} \mathrm{O}_{8+\delta}$ & $\mathrm{b}$ & $(23)$ & 35 & $2500 \pm 300$ & $3200 \pm 300$ & 0.497 \\
\hline $\mathrm{Y}-\mathrm{Bi}_{2} \mathrm{Ca}_{2} \mathrm{SrCu}_{2} \mathrm{O}_{8+\delta}$ & $\mathrm{b}$ & (23) & 40 & $2600 \pm 300$ & $3800 \pm 300$ & 0.418 \\
\hline $\mathrm{Y}-\mathrm{Bi}_{2} \mathrm{Ca}_{2} \mathrm{SrCu}_{2} \mathrm{O}_{8+\delta}$ & $\mathrm{b}$ & $(25)$ & 43 & $4200 \pm 400$ & $5140 \pm 500$ & 0.309 \\
\hline $\mathrm{Tl}_{2} \mathrm{Ba}_{2} \mathrm{CuO}_{6+\delta}$ & $\mathrm{b}$ & $(27)$ & 88 & $5000 \pm 500$ & $6630 \pm 500$ & 0.240 \\
\hline $\mathrm{Nd}_{1.85} \mathrm{Ce}_{0.15} \mathrm{CuO}_{4}$ & $\mathrm{~b}$ & $(10,28)$ & 23 & $28000 \pm 2000$ & $10300 \pm 900$ & 0.154 \\
\hline $\mathrm{Pr}_{1.85} \mathrm{Ce}_{0.15} \mathrm{CuO}_{4}$ & $\mathrm{~b}$ & (13) & 23 & $30000 \pm 3000$ & $10300 \pm 900$ & 0.154 \\
\hline $\mathrm{Pr}_{1.85} \mathrm{Ce}_{0.15} \mathrm{CuO}_{4}$ & $\mathrm{~d}$ & $(29)$ & 21 & $15000 \pm 2000$ & $4820 \pm 600$ & 0.330 \\
\hline $\mathrm{Pr}_{1.87} \mathrm{Ce}_{0.15} \mathrm{CuO}_{4}$ & $\mathrm{~d}$ & (29) & 16 & $50000 \pm 6000$ & $7960 \pm 800$ & 0.207 \\
\hline $\mathrm{La}_{1.87} \mathrm{Sr}_{0.13} \mathrm{CuO}_{4}$ & $\mathrm{~b}$ & $(26)$ & 32 & $7000 \pm 700$ & $5600 \pm 450$ & 0.284 \\
\hline $\mathrm{La}_{1.86} \mathrm{Sr}_{0.14} \mathrm{CuO}_{4}$ & $\mathrm{~b}$ & (26) & 36 & $9000 \pm 900$ & $6000 \pm 500$ & 0.265 \\
\hline $\mathrm{Ba}_{0.62} \mathrm{~K}_{0.38} \mathrm{BiO}_{3}$ & $\mathrm{~b}$ & $(30)$ & 31 & $3800 \pm 300$ & $4240 \pm 400$ & 0.375 \\
\hline $\mathrm{Ba}_{0.60} \mathrm{~K}_{0.40} \mathrm{BiO}_{3}$ & $\mathrm{~b}$ & (30) & 28 & $4400 \pm 300$ & $4240 \pm 400$ & 0.375 \\
\hline $\mathrm{Ba}_{0.54} \mathrm{~K}_{0.46} \mathrm{BiO}_{3}$ & $\mathrm{~b}$ & $(30)$ & 21 & $6000 \pm 300$ & $4240 \pm 400$ & 0.375 \\
\hline $\mathrm{Nb}$ & $\mathrm{d}$ & $(33)$ & 8.3 & $2.5 \mathrm{e} 5$ & 17600 & 0.090 \\
\hline $\mathrm{Nb}$ & $\mathrm{d}$ & $(32)$ & 9.3 & $8.5 \mathrm{e} 5$ & 35800 & 0.044 \\
\hline $\mathrm{Pb}$ & $\mathrm{d}$ & $(32)$ & 7.2 & $1.4 \mathrm{e} 6$ & 41000 & 0.038 \\
\hline
\end{tabular}

${ }^{a}$ Radiation damaged, twinned single crystal.

${ }^{b}$ Light polarized in the $a-b$ plane of a single crystal.

${ }^{c}$ Light polarized along the $a$ axis of a twin-free single crystal.

${ }^{d}$ Thin film or oriented thin film.

nearly two orders of magnitude at low temperatures ${ }^{37}$ This rapid decrease in $1 / \tau$ is also observed optically, but not to the same extent $\frac{38}{3}$ A gap with $d_{x^{2}-y^{2}}$ symmetry may be written as $\Delta_{k}=\Delta_{0}\left[\cos \left(k_{x} a\right)-\cos \left(k_{y} a\right)\right] ;$ the gap reaches a maximum at the $(0, \pi)$ and $(\pi, 0)$ points, and vanishes along the nodal $(\pi, \pi)$ directions. The fact that the scattering rate of the quasiparticles restricted to the nodal regions of the Fermi surface for $T \ll T_{c}$ is quite small has been taken as evidence that these materials are in the clean limit ${ }^{39.40 .41}$ While it is certainly true that for $T \ll T_{c}$ the scattering rate is small and the nodal quasiparticles have very long mean-free paths, it is problematic to assert that the superconductor is therefore in the clean limit. In a normal BCS superconductor $1 / \tau$ is also observed to decrease dramatically below $T_{c}$, regardless of the normal-state value of $1 / \tau$, due to the formation of a condensate ${ }^{42}$ Thus, the criteria of a small value of the quasiparticle scattering rate for $T \ll T_{c}$ is not necessarily a good measure of whether or not the superconductivity is in the clean or dirty limit. As with BCS materials, it is desirable to suppress $T_{c}$ in the copper-oxide materials through the application of a magnetic field to determine the low-temperature behavior of $1 / \tau$. While $\mathrm{H}_{c 2}$ is quite large in the cuprates, experiments using pulsed magnetic fields can suppress $T_{c}$; in these experiments the resistivity of the optimally-doped materials matches the zero-field values at high temperatures due to the low magnetoresistance, and the trend of slowly decreasing resistivity 
continues smoothly to low temperatures, $43,44,45,46,47$ often saturating at a value close to that observed at $T_{c}$. The implication of these experiments is that the normalstate value of $1 / \tau$ is a good measure of the scattering rate in those systems in which $T_{c}$ has been suppressed, and is therefore the value that should be considered when determining whether a system is in the clean or dirty limit. In addition to this explicit approach, a simpler method is to adopt an operational definition which states that if $\rho_{s}$ changes with respect to the normal-state value of $1 / \tau$ then the material is in the dirty limit; when $\rho_{s}$ is no longer sensitive to the value of $1 / \tau$ then the material is in the clean limit. Most of the materials in Fig.2]are studied as a function of carrier doping, but it is also important to note that the introduction of disorder for fixed doping levels has also been studied ${ }^{20}$ The fact that all the observed results follow this linear scaling relation strongly suggests that many of the copper-oxide superconductors are close to or in the dirty limit (i.e., the superfluid density changes in response to variations in $1 / \tau)$.

\section{A. Clean limit}

The BCS model is used to describe the superconductivity of simple metals and alloys. If the normal-state properties may be described by the simple Drude model in which the complex dielectric function is written as $\tilde{\epsilon}(\omega)=\epsilon_{\infty}-\omega_{p}^{2} /[\omega(\omega+i \gamma)]$, where $\omega_{p}^{2}=4 \pi n e^{2} / m^{*}$ is the classical plasma frequency with the free-carrier concentration $n$ and effective mass $m^{*}, \gamma=1 / \tau$ is the scattering rate, and $\epsilon_{\infty}$ is a high-frequency contribution. The dielectric function and the conductivity are related through $\tilde{\sigma}=\sigma_{1}+i \sigma_{2}=-i \omega \tilde{\epsilon} / 4 \pi$, thus the real part of the frequency-dependent conductivity has the form $\sigma_{1}(\omega)=\sigma_{d c} /\left(1+\omega^{2} \tau^{2}\right)$ and $\sigma_{d c}=\omega_{p}^{2} \tau / 4 \pi$, which has the shape of a Lorentzian centered at zero frequency with a width at half-maximum given by $1 / \tau$. The optical conductivity below $T_{c}$ has been calculated from an isotropic ( $s$-wave) energy gap $2 \Delta$ that considers an arbitrary purity level ${ }^{48}$ The clean limit case $(1 / \tau \ll 2 \Delta)$ is illustrated in Fig. 31(a) for the choice $1 / \tau=0.2 \Delta$. An aspect of clean-limit systems is that nearly all of the spectral weight associated with the condensate lies below $2 \Delta$. As a result, the normalized spectral weight of the condensate ${ }^{19}\left(N_{n}-N_{s}\right) / \rho_{s}$ shown in the inset of Fig. 31 (a) approaches unity at frequencies closer to $1 / \tau$ rather than $2 \Delta$. The spectral weight for the condensate (the difference in the area under the two curves, indicated by the hatched region) may be estimated as $\rho_{s} \simeq \sigma_{d c} / \tau$. If $1 / \tau \propto T_{c}$ for $T \simeq T_{c}$ in the copper-oxide materials, 49 then $\rho_{s} \propto \sigma_{d c} T_{c}$, in agreement with the observed scaling relation. It is interesting to note that $1 / \tau \propto T_{c}$ yields rather large values for the normal-state scattering rate, and it has been suggested that the copper-oxide materials are close to the maximum level of dissipation allowed for these systems .50 Furthermore, even though a $d$-wave system complicates the interpretation of the clean and

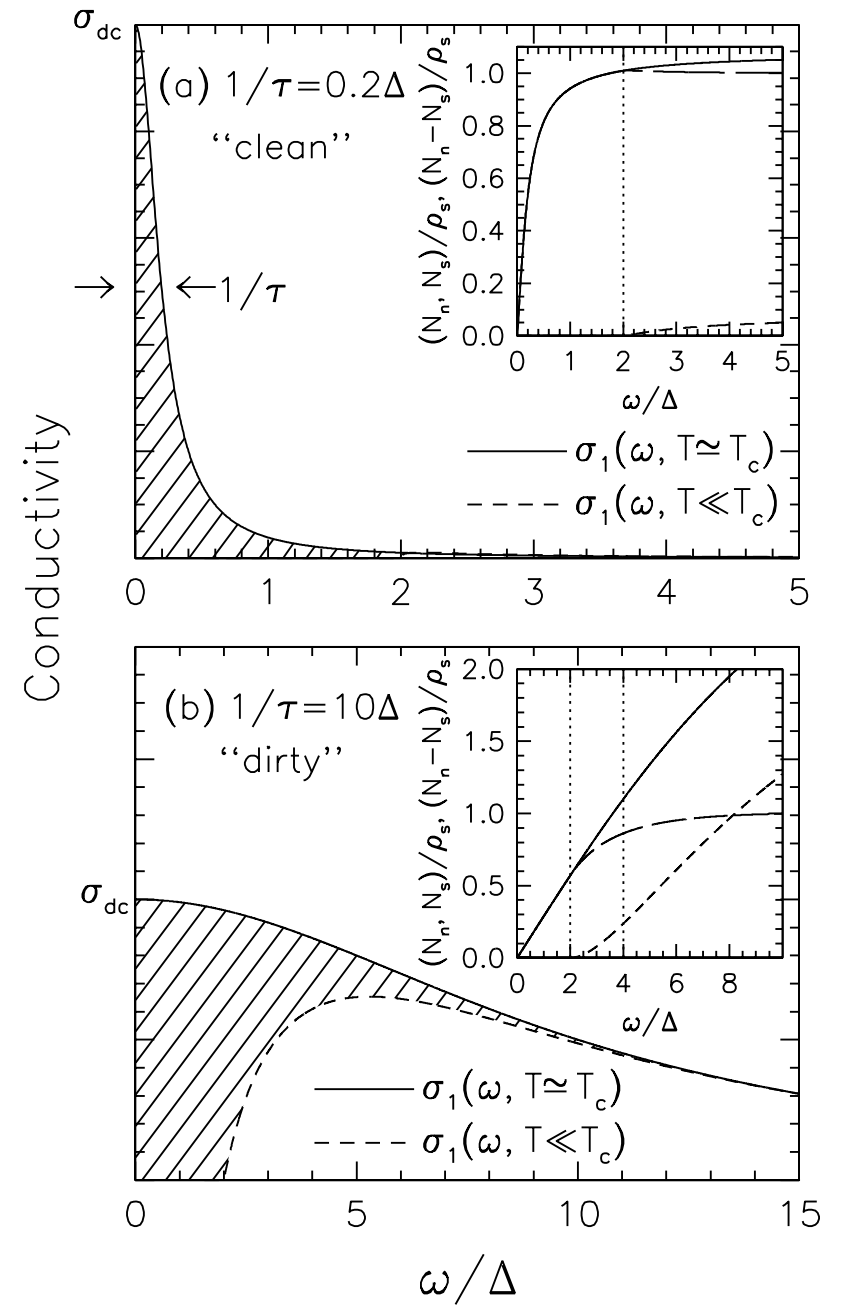

FIG. 3: The optical conductivity for the BCS model in the normal (solid line) and superconducting states (dashed line) for a material in (a) the clean limit $(1 / \tau \ll 2 \Delta)$, and (b) the dirty limit $(1 / \tau>2 \Delta)$. The normal-state conductivity is a Lorentzian centered at zero frequency with a full width at half maximum of $1 / \tau$ for $T \simeq T_{c}$. The spectral weight associated with the formation of a superconducting condensate is indicated by the hatched area. Insets: $N_{n}=N\left(\omega, T \simeq T_{c}\right) / \rho_{s}$ (solid line), $N_{s}=N\left(\omega, T \ll T_{c}\right) / \rho_{s}$ (dashed line), and difference between the two (long-dashed line), normalized with respect to $\rho_{s}$; in the clean limit $\left(N_{n}-N_{s}\right) / \rho_{s}$ converges rapidly to unity, and is fully formed at energies comparable to $1 / \tau$, while in the dirty limit convergence occurs at energies comparable to $4 \Delta$.

dirty limit, large normal-state values of $1 / \tau$ and relatively short normal-state mean-free paths 51 are problematic for a clean-limit picture; to achieve the clean limit it is not only necessary that $1 / \tau \ll 2 \Delta_{0}$, but also that $1 / \tau \lesssim 2 \Delta_{k}$ in the nodal regions. In fact, the clean-limit requirement is much more stringent for a $d$-wave system than it is for a material with an isotropic energy gap, and it is not clear that it will ever be satisfied in the copper-oxide superconductors. This suggests that a dirty-limit view may be more appropriate. 


\section{B. Dirty limit}

In the BCS dirty limit, $1 / \tau>2 \Delta$; this is illustrated in Fig. 3(b) for $1 / \tau=10 \Delta$. In this case the normalstate conductivity is a considerably broader Lorentzian, and much of the spectral weight has been pushed out above $2 \Delta$. As a result, the normalized spectral weight of the condensate, shown in the inset, converges much more slowly than in the clean-limit case. However, a majority of the spectral weight is captured by $2 \Delta$ and $\rho_{s}$ is almost fully formed above $4 \Delta$ (Ref. 19). In the dirty-limit case, the spectral weight of the condensate [the hatched area in Fig. 3(a)] may be estimated as $\rho_{s} \simeq \sigma_{d c} 2 \Delta$. In the BCS model, the energy gap $2 \Delta$ scales linearly with $T_{c}$, yielding $\rho_{s} \propto \sigma_{d c} T_{c}$, which is in agreement with the observed scaling relation. As in the clean-limit case, the nature of the gap is important. If $1 / \tau>2 \Delta_{0}$, the spirit of the dirty-limit case is preserved for all $\Delta_{k}$. While many of the points in Figs. 11 \& 2 are doping-dependent studies and do not track systematic changes in $1 / \tau$, some of these points are for the same chemical doping with different scattering rates resulting from disorder that have either been deliberately introduced, 20 or that exist simply as a byproduct of synthesis (Table I) 52.53 The observation that all the points obey a linear scaling relation satisfies the operational definition of the dirty limit, suggesting that the examined materials are either close to or in the dirty limit.

\section{Behavior of $\mathrm{Nb}$}

It was noted in Fig. 2 that the points for $\mathrm{Nb}$ and $\mathrm{Pb}$ agreed reasonably well with the scaling relation used to describe the copper-oxide superconductors. It is important to determine if these values represent clean or dirtylimit results. The expected behavior of $\mathrm{Nb}$ has been modeled using the BCS mode ${ }^{48}$ for an arbitrary purity level with a critical temperature of $T_{c}=9.2 \mathrm{~K}$ and a gap of $2 \Delta=22.3 \mathrm{~cm}^{-1}$ (the BCS weak-coupling limit $\left.2 \Delta=3.5 k_{B} T_{c}\right)$. The normal-state is described using the Drude model with a classical plasma frequency of $\omega_{p}=56000 \mathrm{~cm}^{-1}$ (Ref. 55) and a range of scattering rates $1 / \tau=0.05 \Delta \rightarrow 50 \Delta$; from the Drude model the dc conductivity is $\sigma_{d c}=\omega_{p}^{2} \tau / 60$ (in units of $\Omega^{-1} \mathrm{~cm}^{-1}$ when the plasma frequency and the scattering rate have units of $\left.\mathrm{cm}^{-1}\right)$. The spectral weight of the condensate $\rho_{s}=N_{n}-N_{s}$ has been determined by integrating to $\omega \simeq 200 \Delta$, where $\rho_{s}$ is observed to converge for all the values of $1 / \tau$ examined. The result of this calculation is shown as the solid line in Fig. 4, and the vertical dashed line indicates where $1 / \tau=2 \Delta$. The point to the right of the dashed line is for $\mathrm{Nb}$ recrystallized in ultra-high vacuum 54 to achieve clean-limit conditions in which the residual resistivity ratios $\left[\rho(\mathrm{RT}) / \rho\left(T \gtrsim T_{c}\right)\right]$ are well in excess of 100 , and where $\rho_{s} \rightarrow \omega_{p}^{2}$ for $T \ll T_{c}$. As the scattering rate increases and the material becomes progressively more "dirty", the strength of the condensate

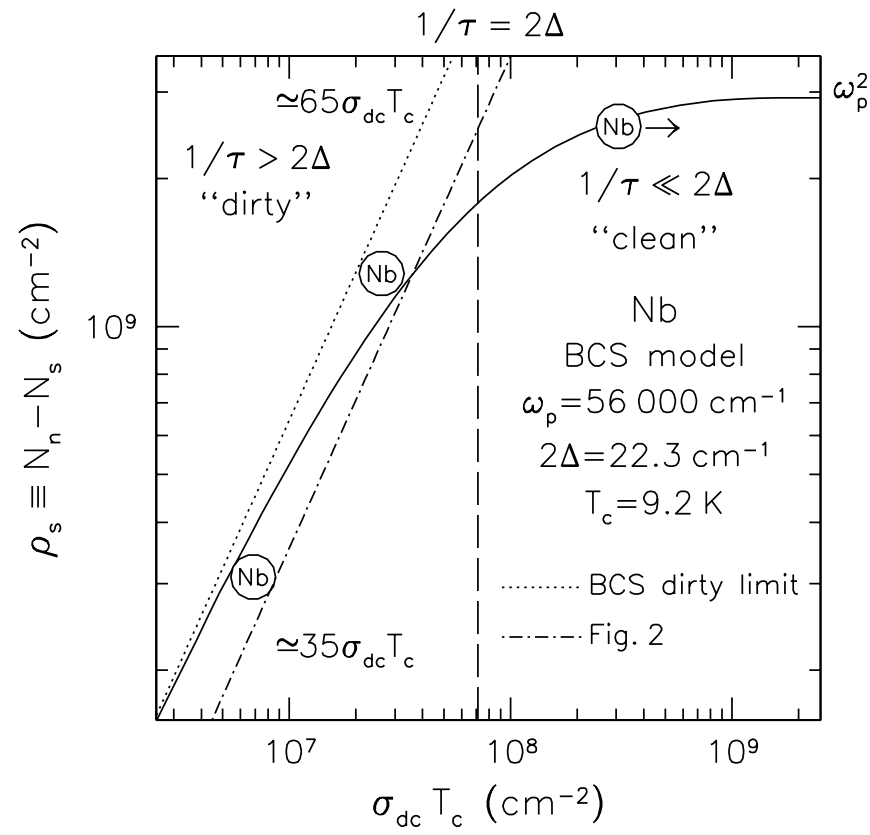

FIG. 4: The log-log plot of the predicted behavior from the BCS model of the strength of the condensate for $\mathrm{Nb}$ for a wide range of scattering rates $1 / \tau=0.05 \Delta \rightarrow 50 \Delta$, and assuming a plasma frequency $\omega_{p}=56000 \mathrm{~cm}^{-1}$, critical temperature $T_{c}=9.2 \mathrm{~K}$ and an energy gap of $2 \Delta=3.5 k_{B} T_{c}$ (solid line). The dashed line indicates $1 / \tau=2 \Delta$. To the right of this line the material approaches the clean limit with a residual resistance ratio (RRR) of $\gtrsim 100$; the right arrow indicates that for larger RRR's, $\sigma_{d c}$ close to $T_{c}$ increases, but $\rho_{s}$ has saturated to $\omega_{p}^{2}$ (the data point for $\mathrm{Nb}$ in this regime is from Ref. 54). As the scattering rate increases, the strength of the condensate adopts a linear scaling behavior (dotted line); the two points for Nb (Refs. 32 33) shown in Fig. 2lie close to this line, indicating that they are in the dirty limit. The scaling relation shown in Fig. 2 (dash-dot line) is slightly offset from the BCS dirty-limit result.

begins to decrease until it adopts the linear scaling behavior $\rho_{s} \simeq 65 \sigma_{d c} T_{c}$ observed in Fig. 4. (It should be noted that the BCS model yields the same asymptotic behavior in the dirty limit, regardless of the choice of $\omega_{p}$ or $\Delta$; the constant is only sensitive upon the ratio of $\Delta$ to $T_{c}$.) The two points for $\mathrm{Nb}$ shown in Fig. 2 (reproduced in Fig. 4), fall close to this line $\mathrm{e}^{32,33}$ and are clearly in the dirty limit. Thus, the scaling relation $\rho_{s} \propto \sigma_{d c} T_{c}$ is the hallmark of a BCS dirty-limit system 56 The scaling relation for the copper-oxide superconductors $\rho_{s} \simeq 35 \sigma_{d c} T_{c}$ is somewhat less than the $\rho_{s} \simeq 65 \sigma_{d c} T_{c}$ asymptotic behavior observed for the weak-limit BCS material. However, in the log-log representation of Fig. 2 the numerical constant in the scaling relation is the offset of the line. The line may be shifted by assuming different ratios between $2 \Delta$ and $k_{B} T_{c}$; the initial value of $\simeq 65$ was based on the weak-coupling value of $2 \Delta / k_{B} T_{c} \simeq 3.5$, while the observed value of $\simeq 35$ may be reproduced by assuming a smaller ratio $2 \Delta / k_{B} T_{c} \simeq 2$. The difference may also arise from the different symmetry of the superconducting energy gap in the two systems, and the fact that in the 
copper-oxide materials there is still a substantial amount of low-frequency residual conductivity at low temperature. Regardless of these differences, the empirical scaling relation $\rho_{s} \propto \sigma_{d c} T_{c}$ is observed in both the copper oxide and disordered elemental superconductors. If it is true in general that $\rho_{s} \propto \sigma_{d c} 2 \Delta$, then this necessarily implies that $\Delta \propto T_{c}$. In the optimally-doped and overdoped materials, there is some evidence that $\Delta_{0} \propto T_{c}$ (Refs. 57.58). In the underdoped materials, large gaps are observed to develop in the normal state ${ }^{59}$ well above $T_{c}$. While it has been noted that the energy scale over which spectral weight is transferred into the condensate is much larger in the underdoped materials than it is for the optimally-doped materials,,$\frac{60.61}{6}$ the majority of the spectral weight is still captured at energies comparable to $T_{c}$. This would tend to support the view that the energy scale relevant to phase coherence and the formation of the condensate is proportional to $T_{c}$.

It is of some interest at this point to compare the empirical relation, that $\rho_{s}$ is proportional to $\sigma_{d c} T_{c}$, with the expression for the penetration depth that is given by the Ginzburg-Landau theory modified for the dirty limit. In general, the expression for the London penetration depth is given by $\lambda_{L}(T \rightarrow 0)=\sqrt{m c^{2} /\left(4 \pi n_{s} e^{2}\right)}$, where $n_{s} \equiv n$ is the superconducting carrier concentration. In the dirty limit one can show that $\rho_{s}($ dirty $) / \rho_{s}($ clean $)=$ $l / \xi_{0}$ (Ref. 31). An increase in $1 / \tau$ reduces the amount of superfluid and the penetration depth increases and can be written as $\lambda^{2}=\left(\xi_{0} / l\right) \lambda_{L}^{2}$. Since $\lambda^{2} \propto 1 / \rho_{s}$, $\xi_{0} \propto 1 / T_{c}$, and $\sigma_{d c} \propto l$, then one can recover the result that $\rho_{s} \propto \sigma_{d c} T_{c}$. It is possible that in a $d$-wave system the presence of nodal regions with a small superfluid density and $\Delta_{k} \ll \Delta_{0}$, that the coherence length in the above expression for $\lambda^{2}$ now involves some average including the nodal regions.

\section{The c axis}

It was previously observed ${ }^{13}$ that the scaling relation $\rho_{s} \simeq 35 \sigma_{d c} T_{c}$ is a universal result that describes not only the the $a-b$ planes, but the $c$ axis as well, as shown in Fig. 5] While a description of the scaling based scattering rates within the context of clean and dirty limits may be appropriate for the $a-b$ planes where the transport is coherent, it is inappropriate along the $c$ axis, where the activated nature of the temperature dependence of the resistivity indicates that the transport in this direction is incoherent and governed by hopping. ${ }^{63}$ In this case, the superconductivity along the $c$ axis may be described by the Josephson effect, which for the BCS weak coupling case $\left(2 \Delta=3.5 k_{B} T_{c}\right)$ yields $\rho_{s} \simeq 65 \sigma_{d c} T_{c}$ (Ref. 62). Surprisingly, this is precisely the result that was obtained in the $a-b$ planes for the BCS weak-coupling case in the dirty limit in Fig. 4 indicating that from a functional point of view the dirty limit and the Josephson effect are nearly identical. One interpretation of this result is that the Josephson effect may arise naturally out of sys-

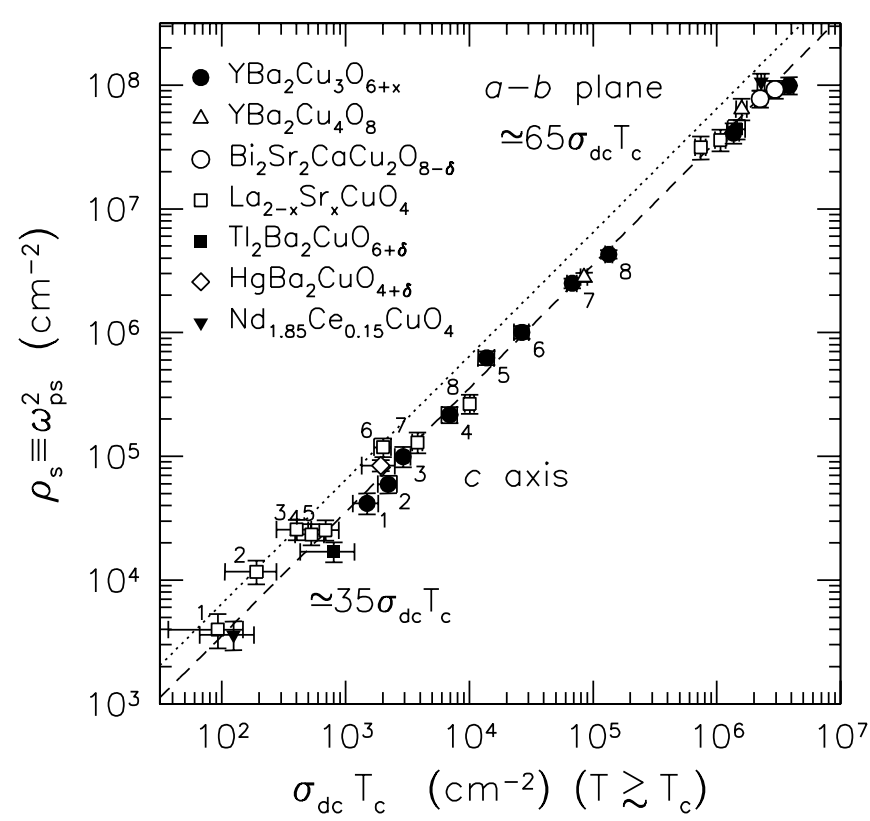

FIG. 5: The log-log plot of the superfluid density expressed as a plasma frequency $\rho_{s} \equiv \omega_{p S}^{2}$ vs $\sigma_{d c} T_{c}$ for the $a$ - $b$ planes and the $c$ axis for a variety of cuprates. Within error, all of the points fall on the same universal (dashed) line defined by $\rho_{s} \simeq 35 \sigma_{d c} T_{c}$; the dotted line is the dirty limit result $\rho_{s} \simeq$ $65 \sigma_{d c} T_{c}$ for the BCS weak-coupling case $\left(2 \Delta=3.5 k_{B} T_{c}\right)$ from Fig. 4 and also represents the Josephson result for the BCS weak-coupling case, used to describe the scaling along the $c$-axis. ${ }^{62}$ (Values for the $c$-axis points are listed in the supplemental infomation of Ref. 13.)

tems with an increasing amount of disorder, and that as a result any crossover from coherent to incoherent behavior results in the same overall scaling relation. Another somewhat more speculative possibility is that the copperoxide superconductors may be so electronically inhomogeneous that it may be possible to view the Josephson effect as appropriate not only for the $c$ axis, but for the $a-b$ planes as well 64.65

\section{CONCLUSIONS}

The implications of the linear scaling relation $\rho_{s} \propto$ $\sigma_{d c} T_{c}$ in the copper-oxide superconductors has been examined within the context of clean and dirty-limit systems. In the conventional BCS superconductors (such as $\mathrm{Nb}$ ), this linear scaling is the hallmark of a dirty-limit system. The copper-oxide materials are thought to be $d$ wave superconductors, in which the clean limit is difficult to achieve. The observed linear scaling strongly suggests that the copper-oxide superconductors are either close to or in the dirty limit. Estimates of $\rho_{s}$ based on geometric arguments imply that the energy scale below which the majority of the spectral weight is transferred into the condensate scales linearly with $T_{c}$. The $a-b$ planes and the $c$ axis follow the same scaling relation 13 The scaling behavior for the dirty limit and the Josephson 
effect (assuming a BCS formalism) is essentially identical from a functional point of view, suggesting that in some regime the dirty limit and the Josephson effect may be viewed as equivalent. This raises the possibility that electronic inhomogeneities may play an important role in the mechanism of superconductivity in the copper-oxide high-temperature superconductors.

\section{Acknowledgments}

The authors would like to thank Y. Ando, D. N. Basov, D. A. Bonn, I. Bozovic, A. V. Chubukov, M. Greven,
W. N. Hardy, P. D. Johnson, S. A. Kivelson, P. A. Lee, T. M. Rice, D. B. Tanner, T. Timusk, and J. J. Tu for useful discussions. Work at Brookhaven was supported by the DOE under contract number DE-AC02-98CH10886.
* Electronic address: homes@bnl.gov

1 R. J. Whittaker, Nature 401, 865 (1999).

2 J. H. Brown and G. B. West, eds., Scaling in biology (Oxford University Press, Oxford, 1999).

3 J. G. Bednorz and K. A. Mueller, Z. Phys. B 64, 189 (1986).

4 T. Schneider, Europhys. Lett. 60, 141 (2002).

${ }^{5}$ Y. J. Uemura, G. M. Luke, B. J. Sternlieb, J. H. Brewer, J. F. Carolan, W. N. Hardy, R. Kadono, R. F. Kiefl, S. R. Kreitzman, P. Mulhern, et al., Phys. Rev. Lett. 62, 2317 (1989).

${ }^{6}$ Y. J. Uemura, L. P. Le, G. M. Luke, B. J. Sternlieb, W. D. Wu, J. H. Brewer, T. M. Riseman, C. L. Seaman, M. B. Maple, M. Ishikawa, et al., Phys. Rev. Lett. 66, 2665 (1991).

7 Y. Zuev, M. S. Kim, and T. R. Lemberger, condmat/0410135.

8 C. Niedermayer, C. Bernhard, U. Binninger, H. Glckler, J. L. Tallon, E. J. Ansaldo, and J. I. Budnick, Phys. Rev. Lett. 71, 17641767 (1993).

9 J. L. Tallon, J. W. Loram, J. R. Cooper, C. Panagopoulos, and C. Bernhard, 68, 180501 (2003).

10 C. C. Homes, B. P. Clayman, J. L. Peng, and R. L. Greene, Phys. Rev. B 56, 5525 (1997).

11 C. C. Homes, B. P. Clayman, J. L. Peng, and R. L. Greene, J. Phys. Chem. Solids 59, 1979 (1998).

12 A. Pimenov, A. Loidl, B. Schey, B. Stritzker, G. Jakob, H. Adrian, A. V. Pronin, and Y. G. Goncharov, Europhys. Lett. 48, 73 (1999).

13 C. C. Homes, S. V. Dordevic, M. Strongin, D. A. Bonn, R. Liang, W. N. Hardy, S. Komiya, Y. Ando, G. Yu, N. Kaneko, et al., Nature 430, 539 (2004).

14 J. L. Tallon, J. R. Cooper, S. H. Naqib, and J. W. Loram, cond-mat/0410568.

15 C. C. Homes, M. Reedyk, D. Crandles, and T. Timusk, Appl. Opt. 32, 2972 (1993).

16 The term $120 / \pi$ before the integral is the result of both a normalization of the integral (a geometric term) and a conversion of units, where it is assumed that the units of conductivity are in $\Omega^{-1} \mathrm{~cm}^{-1}$, and that the frequency is in $\mathrm{cm}^{-1}$, so that the integral yields $\mathrm{cm}^{-2}$. To recast all the units into $\mathrm{cm}^{-1}$, two other unit conversions are implicitly employed in the text; $1 \mathrm{~cm}^{-1}=0.21 \Omega^{-1} \mathrm{~cm}^{-1}$ and $1 \mathrm{~cm}^{-1}=1.44 \mathrm{~K}$.

17 R. A. Ferrell and R. E. Glover, III, Phys. Rev. 109, 1398
(1958).

18 M. Tinkham and R. A. Ferrell, Phys. Rev. Lett. 2, 331 (1959).

19 D. N. Basov, S. I. Woods, A. S. Katz, E. J. Singley, R. C. Dynes, M. Xu, D. G. Hinks, C. C. Homes, and M. Strongin, Science 283, 49 (1999).

20 D. N. Basov, A. V. Puchkov, R. A. Hughes, T. Strach, J. Preston, T. Timusk, D. A. Bonn, R. Liang, and W. N. Hardy, Phys. Rev. B 49, 12165 (1994).

21 D. N. Basov, R. Liang, D. A. Bonn, W. N. Hardy, B. Dabrowski, M. Quijada, D. B. Tanner, J. P. Rice, D. M. Ginsberg, and T. Timusk, Phys. Rev. Lett. 74, 598 (1995).

22 C. C. Homes, D. A. Bonn, R. Liang, W. N. Hardy, D. N. Basov, T. Timusk, and B. P. Clayman, Phys. Rev. B 60, 9782 (1999).

23 H. L. Liu, M. A. Quijada, A. M. Zibold, Y.-D. Yoon, D. B. Tanner, G. Cao, J. E. Crow, H. Berger, G. Margaritondo, L. Forro, et al., J. Phys: Condens. Matter 11, 239 (1990).

24 J. J. Tu, C. C. Homes, G. D. Gu, D. N. Basov, and M. Strongin, Phys. Rev. B 66, 144514 (2002).

25 N. L. Wang, A. W. McConnell, and B. P. Clayman, Phys. Rev. B 60, 14883 (1999).

26 T. Startseva, T. Timusk, A. V. Puchkov, D. N. Basov, H. A. Mook, M. Okuya, T. Kimura, and K. Kishio, Phys. Rev. B 59, 7184 (1999).

27 A. V. Puchkov, T. Timusk, S. Doyle, and A. M. Herman, Phys. Rev. B 51, 3312 (1995).

28 E. J. Singley, D. N. Basov, K. Kurahashi, T. Uefuji, and K. Yamada, Phys. Rev. B 64, 224503 (2001).

29 A. Zimmers, R. Lobo, N. Bontemps, C. Homes, M. Barr, Y. Dagan, and R. Greene, Phys. Rev. B 70, 132502 (2004).

30 A. V. Puchkov, T. Timusk, M. A. Karlow, S. L. Cooper, P. D. Han, and D. A. Payne, Phys. Rev. B 54, 6686 (1996).

31 R. A. Ferrell and H. Schmidt, Phys. Lett. 25A, 544 (1967).

32 O. Klein, E. J. Nicol, K. Holczer, and G. Grüner, Phys. Rev. B 50, 6307 (1994).

33 A. V. Pronin, M. Dressel, A. Pimonev, A. Loidl, I. V. Roshchin, and L. H. Greene, Phys. Rev. B 57, 14416 (1998).

34 Z. Shen, D. S. Dessau, B. O. Wells, D. M. King, W. E. Spicer, A. J. Arko, D. Marshall, L. W. Lombardo, A. Kapitulnik, P. Dickinson, et al., Phys. Rev. Lett. 70, 1553 (1993).

35 W. N. Hardy, D. A. Bonn, D. C. Morgan, R. Liang, and K. Zhang, Phys. Rev. Lett. 70, 3999 (1993). 
36 Y. Iye, in Physical Properties of High-Temperature Superconductors III, edited by D. M. Ginsberg (World Scientific, Singapore, 1992), pp. 285-361.

37 D. A. Bonn, R. Liang, T. M. Riseman, D. J. Baar, D. C. Morgan, K. Zhang, P. Dosanjh, T. L. Duty, A. MacFarlane, G. D. Morris, et al., Phys. Rev. B 47, 11314 (1993).

38 A. V. Puchkov, D. N. Basov, and T. Timusk, J. Phys: Condens. Matter 8, 10049 (1996).

39 M. Sutherland, D. G. Hawthorn, R. W. Hill, R. Ronning, S. Wakimoto, H. Zhang, C. Proust, E. Boaknin, C. Lupien, L. Taillefer, et al., Phys. Rev. B 67, 174520 (2003).

${ }^{40}$ P. J. Turner, R. Harris, S. Kamal, M. E. Hayden, D. M. Broun, D. C. Morgan, A. Hosseini, P. Dosanjh, G. K. Mullins, J. S. Preston, et al., Phys. Rev. Lett. 90, 237005 (2003).

41 R. W. Hill, C. Lupien, M. Sutherland, E. Boaknin, D. G. Hawthorn, C. Proust, F. Ronning, L. Taillefer, R. Liang, D. A. Bonn, et al., Phys. Rev. Lett. 92, 027001 (2004).

42 D. N. Basov, E. J. Singley, and S. V. Dordevic, Phys. Rev. B 65, 054516 (2002).

${ }^{43}$ G. S. Boebinger, Y. Ando, A. Passner, T. Kimura, M. Okura, J. Shimoyama, K. Kishio, K. Tamasaku, N. Ichikawa, and S. Uchida, Phys. Rev. Lett. 77, 5417 (1996).

44 Y. Ando, G. S. Boebinger, A. Passner, N. L. Wang, C. Geibel, and F. Steglich, Phys. Rev. Lett. 77, 2065 (1996).

45 Y. Ando, G. S. Boebinger, A. Passner, N. L. Wang, C. Geibel, and F. Steglich, Phys. Rev. Lett. 79, 2595 (1997).

46 Y. Ando, G. S. Boebinger, A. Passner, N. L. Wang, C. Geibel, F. Steglich, I. E. Trofimov, and F. F. Balakirev, Phys. Rev. B 56, R8530 (1997).

47 S. Ono, Y. Ando, T. Murayama, F. F. Balakirev, J. B. Betts, and G. S. Boebinger, Phys. Rev. Lett. 85, 638 (2000).

48 W. Zimmerman, E. H. Brandt, M. Bauer, E. Seider, and L. Genzel, Physica C 183, 99 (1991).

49 J. Orenstein, G. A. Thomas, A. J. Millis, S. L. Cooper, D. H. Rapkine, T. Timusk, L. F. Schneemeyer, and J. V. Waszczak, Phys. Rev. B 42, 6342 (1990).

50 J. Zaanen, Nature 430, 512 (2004).

51 Y.-S. Lee, K. Segawa, Y. Ando, and D. N. Basov, Phys.
Rev. B 70, 014518 (2004).

${ }^{52}$ W. N. Hardy, S. Kamal, D. A. Bonn, K. Zhang, R. Liang, E. Klein, D. C. Morgan, and D. Baar, Physica B 197, 609 (1994).

53 A. Hosseini, R. Harris, S. Kamal, P. Dosanjh, J. Preston, R. Liang, W. N. Hardy, and D. A. Bonn, Phys. Rev. B 60, 1349 (1999).

54 C. Varmazis and M. Strongin, Phys. Rev. B 10, 1885 (1974).

55 E. D. Palik, ed., Handbook of Optical Constants of Solids II (Academic Press, San Diego, 1998).

56 R. A. Smith and V. Ambegaokar, Phys. Rev. B 45, 2463 (1992), in the absence of weak localization, in the dirty limit $\Gamma \gg \Delta$, it was shown that $\rho_{s}=4 \pi^{2} \sigma_{d c} \Delta \tanh [\Delta / 2 T]$, where $\sigma_{d c}$ is the dc conductivity in the normal state and $\Delta$ is the superconducting energy gap. Assuming that the gap scales with $T_{c}$ and that $T \ll T_{c}$, then $\rho_{s} \propto \sigma_{d c} T_{c}$. This result is similar to the one obtained in the text using arguments based on the spectral weight.

57 C. Panagopoulos and T. Xiang, Phys. Rev. Lett. 81, 2336 (1998).

58 A. Damascelli, Z. Hussain, and Z.-X. Shen, Rev. Mod. Phys. 75, 473 (2003).

59 N.-C. Yeh, C.-T. Chen, G. Hammerl, J. Mannhart, A. Schmehl, C. W. Schneider, R. R. Schulz, S. Tajima, K. Yoshida, D. Garrigus, et al., Phys. Rev. Lett. 87, 087003 (2001).

60 A. F. Santander-Syro, R. P. S. M. Lobo, N. Bontemps, Z. Konstantinovic, Z. Li, and H. Raffy, Phys. Rev. Lett 88, 097005 (2002).

61 C. C. Homes, S. V. Dordevic, D. A. Bonn, R. Liang, and W. N. Hardy, Phys. Rev. B 69, 024514 (2004).

62 C. C. Homes, S. V. Dordevic, D. A. Bonn, R. Liang, W. N. Hardy, and T. Timusk, cond-mat/0312211.

63 S. L. Cooper and K. E. Gray, in Physical Properties of High-Temperature Superconductors IV, edited by D. M. Ginsberg (World Scientific, Singapore, 1994), pp. 61-188.

64 J. Jung, H. Yan, H. Darhmaoui, and W.-K. Kwok, in Superconducting and Related Oxides: Physics and Nanoengineering III, edited by D. Pavuna and I. Bozovic (SPIE, Washington, 1998), vol. 3481, pp. 172-181.

65 J. J. Tu, M. Strongin, and Y. Imry, cond-mat/0405625. 